\title{
Estudio comparativo de los componentes del balance hídrico en un bosque nativo y una pradera en el sur de Chile
}

\author{
Comparative study of water balance components in a native forest and \\ a meadow in southern Chile
}

\author{
Cristian Echeverría ${ }^{a}$ b* $^{*}$, Anton Huber ${ }^{\mathrm{c}}$, Florent Taberlet ${ }^{\mathrm{d}}$ \\ *Autor de correspondencia: ${ }^{a}$ Universidad de Concepción, Instituto de Manejo Forestal y Medio Ambiente, \\ Casilla 160-C, Concepción, Chile, Tel.: (56-41) 2204936, fax: (56-41) 2255164, cristian.echeverria@udec.cl

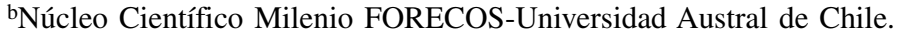 \\ cUniversidad Austral de Chile, Instituto de Geociencias. Casilla 567, Valdivia, Chile. \\ dEcole Centrale Paris, 92295 Châtenay-Malabry, Francia.
}

\begin{abstract}
SUMMARY
A comparative study of water balance was conducted in a roble-olivillo forest and a natural meadow in southern Chile. We analysed the different components of precipitation redistribution, evapotranspiration, percolation and changes in soil water content until $3 \mathrm{~m}$ of depth. As related to a total precipitation of 3,024 mm, evapotranspiration and percolation were $13 \%$ and $87 \%$ for the meadow, and $33 \%$ and $66 \%$ for the forest respectively. Soil water reserves varied down to $300 \mathrm{~cm}$ of depth in the soil of the forest while in the meadow this situation was recorded only to a depth of $1 \mathrm{~m}$. It can be concluded that higher water consumption in the forest and higher percolation water in the meadow were ascribed mainly to the difference in development of both root systems and the canopy characteristic of each of these vegetation covers.
\end{abstract}

Key words: evapotranspiration, precipitation redistribution, soil water, olivillo, roble.

\section{RESUMEN}

Se realizó un estudio comparativo del balance hídrico entre un bosque de roble-olivillo y una pradera natural en la zona de Valdivia, sur de Chile. Para ello se determinaron los diferentes componentes de la redistribución de las precipitaciones, evapotranspiración, percolación y la variación del contenido de agua en el suelo hasta los $3 \mathrm{~m}$ de profundidad. Las precipitaciones del período de estudio fueron de $3.024 \mathrm{~mm}$, de las cuales el 13\% en la pradera y $33 \%$ en el bosque se reintegraron a la atmósfera por evapotranspiración. La percolación para las mismas cubiertas vegetales fue de un $87 \%$ y $66 \%$, respectivamente. La reserva de agua edáfica durante el estío varió en todo el perfil considerado en el bosque y aproximadamente hasta $1 \mathrm{~m}$ en la pradera. En comparación con la pradera, en el bosque se observó un consumo de agua superior por evapotranspiración y uno menor por percolación debido a la mayor profundidad que alcanza su sistema radicular y a las superiores pérdidas por intercepción.

Palabras clave: evapotranspiración, redistribución precipitaciones, agua edáfica, olivillo, roble.

\section{INTRODUCCIÓN}

El ciclo hidrológico es un modelo de circulación general que implica un despliegue complejo de los movimientos y transformaciones del agua (Lee 1980). Es sabido que las diversas cubiertas vegetales están íntimamente relacionadas con la cuantía, distribución y posibilidades de aprovechamiento de los recursos hídricos. Los cambios antrópicos o naturales que se producen en los ecosistemas pueden tener repercusiones considerables sobre el balance hídrico local (Ward y Trimble 2004). Aussenac y Boulangeat (1980) reconocen que las influencias de los bosques sobre el ciclo hídrico son complejas y variables según el tipo de clima.
Esto se debe a que la cubierta vegetal, particularmente los árboles, juega un papel trascendental en la redistribución de las precipitaciones, ya que ellos modifican la cantidad y lugar donde alcanzan la superficie (Hewlett y Nutter 1969, López y Blanco 1976, Daniel et al. 1982, Spurr y Barnes 1982, Huber y Oyarzún 1990).

Al producirse una precipitación pluvial o nival sobre un bosque, una parte de ésta es interceptada por su follaje (Hewlett 1969, Grimm y Fassbender 1981). Esta agua, capturada por el dosel de la vegetación, se puede redistribuir a través del follaje (precipitación directa), escurrir por los troncos (escurrimiento fustal) o evaporar desde las hojas (pérdidas por intercepción) (López y Blanco 1978, Lee 
1980, Crockford y Richardson 1990). Las precipitaciones que alcanzan el suelo y penetran en él modifican la reserva del agua edáfica, son utilizadas en la evapotranspiración o percolan hacia los estratos inferiores del suelo.

La intervención silvícola de un bosque o su reemplazo por otro tipo de cubierta vegetal induce alteraciones en los componentes de la redistribución de las precipitaciones, origina variaciones en las reservas de agua del suelo y en los montos de agua involucrados en la evapotranspiración y percolación (Ward y Trimble 2004, Knoche 2005, Neira 2005, Thanapakpawin et al. 2006, Zimmermann et al. 2006). En particular, los bosques templados de Chile han estado sometidos a continuos procesos de degradación y reemplazo por otros usos del suelo (Echeverría et al. 2006). Sin embargo, muy poco se sabe de las consecuencias que dicha transformación tiene sobre los componentes hidrológicos. Ante tal eventualidad es necesario conocer lo mejor posible estos ecosistemas para predecir este tipo de consecuencias.

En el presente estudio se determinan los diferentes componentes del balance hídrico de un bosque de robleolivillo (Nothofagus obliqua (Mirb.) Oerst.-Aextoxicon punctatum Ruiz et Pavón) y sus resultados se comparan con los de una pradera natural en el sur de Chile. Debido a las distintas características de ambas cubiertas vegetales, se espera que el bosque tenga una mayor evapotranspiración y por consiguiente una menor percolación que la pradera.

\section{MÉTODOS}

La investigación se llevó a cabo en el Fundo San Martín, ubicado a $25 \mathrm{~km}$ al noroeste de la ciudad de Valdivia (38 $48^{\prime}$ 'S y 7314' O, 10 m s.n.m.). El área posee una topografía prácticamente plana con pendientes inferiores al 5\%. La zona tiene un clima oceánico con influencia mediterránea. Las precipitaciones caen durante todo el año con un promedio anual de $2.400 \mathrm{~mm}$. Entre los meses de abril y septiembre precipita una cantidad equivalente al $75 \%$ del total anual. El mes más seco es enero con 60 mm y el más húmedo, julio con 400 mm (Huber 1970). El suelo de la zona pertenece a la serie Valdivia (IREN et al. 1978), del tipo trumao aluvial, cuyos suelos son derivados de sedimentos de cenizas depositadas a consecuencia de erupciones volcánicas (Weinberger y Binsack 1970). En su horizonte inferior el suelo se encuentra mezclado con materiales fluvioglaciales más antiguos.

El bosque nativo corresponde a un ecotono entre el bosque de Roble-Laurel-Lingue (Nothofago-PerceetumLinguae) y una subasociación del bosque de olivillo (Lapagerio-Aextoxiconetum-Rigodietosum (Steubing et al. 1979). El estrato arbóreo superior está integrado por Nothofagus obliqua y Eucryphia cordifolia Cav., y alcanza una altura de hasta $45 \mathrm{~m}$. El estrato arbóreo medio está conformado por Aextoxicon punctatum, Laureliopsis philippiana (Looser) Schodde., Laurelia sempervirens (Ruiz et Pavón) Tul. y Podocarpus salignus D. Don. con una altura entre los 15 y $30 \mathrm{~m}$, mientras que el estrato arbóreo inferior, ubicado entre los 10 y $15 \mathrm{~m}$, está compuesto por Drimys winteri J. R. et Forster., Gevuina avellana Mol., Amomyrtus luma (Mol.) Legrand et Kausel. y Luma apiculata (DC.) Burret. La cobertura media del dosel arbóreo es de un 94\%. El estrato arbustivo está integrado principalmente por Rhaphithamnus spinosus (Juss.) Mold. y Chusquea quila Kunth. El suelo está cubierto por una capa de hojarasca de aproximadamente $3 \mathrm{~cm}$ de espesor y por Rigodium implexum Kunze ex Schwaegr.

Como superficie de comparación se utilizó una pradera natural rala y de poco desarrollo. La pradera corresponde a una franja de alrededor de $50 \mathrm{~m}$ de ancho que cruza el bosque, constituida principalmente por chépica (Agrostis capillaris L.) y pasto miel (Holcus lanatus L.). La profundidad de enraizamiento alcanza aproximadamente los $30 \mathrm{~cm}$. La pradera no tiene ningún uso ganadero.

Para el estudio se delimitó una superficie de aproximadamente 100 x $100 \mathrm{~m}$ en el bosque y 30 x $30 \mathrm{~m}$ en la pradera, en los cuales se instalaron los equipos de medición (figura 1).

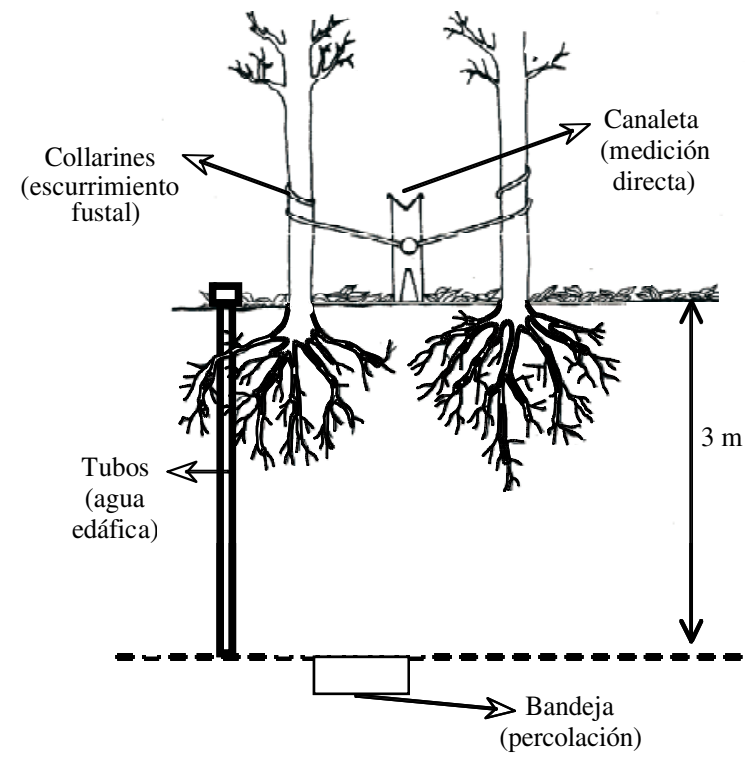

Figura 1. Diseño del ensayo para medición de los componentes del balance hídrico en el bosque de roble-olivillo y la pradera.

Design of the study for the measurement of the water balance components in the roble-olivillo forest and the meadow.

La cantidad de agua involucrada en la evapotranspiración se calculó con la ecuación del balance hídrico [1], basada en la metodología de continuidad de masas propuesta por Feller (1981):

$$
\mathrm{EVPT}=\mathrm{PP}-\mathrm{IC}-\Delta \mathrm{W}-\mathrm{A}-\mathrm{PER}
$$

donde: EVPT es la evapotranspiración ( $\mathrm{mm})$; PP es la precipitación incidente $(\mathrm{mm})$; IC pérdidas de agua por 
intercepción del dosel; A escorrentía superficial; $\Delta \mathrm{W}$ es la variación del contenido de agua del suelo $(\mathrm{mm})$; PER es la percolación (mm).

La precipitación incidente se midió con dos pluviógrafos Wilhellambrecht con precisión de $0,1 \mathrm{~mm}$ ubicados en la pradera. Las pérdidas de agua por intercepción (IC) se calcularon de acuerdo con la ecuación siguiente:

$$
\mathrm{IC}=\mathrm{PP}-(\mathrm{PD}+\mathrm{PF})
$$

donde: IC es la pérdida de agua por intercepción del dosel $(\mathrm{mm})$; PD es la precipitación directa $(\mathrm{mm})$ y PF es el escurrimiento fustal (mm).

Con el fin de conocer la variabilidad de las precipitaciones del año de medición con respecto a un año promedio, la precipitación incidente registrada durante la investigación fue comparada con la precipitación promedio mensual de los últimos 25 años. Ambos valores de precipitación fueron obtenidos en la estación pluviométrica en el Campus Isla Teja de la Universidad Austral de Chile. Esta estación se ubica aproximadamente a $10 \mathrm{~km}$ en línea recta del área de estudio.

La precipitación directa se midió con una canaleta metálica en forma de $\mathrm{V}$ de $15 \mathrm{~cm}$ de ancho y $50 \mathrm{~m}$ de largo (superficie de recolección de $7,5 \mathrm{~m}^{2}$ equivalente a 375 pluviómetros), ubicada a $30 \mathrm{~cm}$ sobre el suelo. El escurrimiento fustal se midió con collarines de goma sellados alrededor del tronco de todos los árboles, que estaban ubicados a ambos lados de la canaleta hasta una distancia de $10 \mathrm{~m}$ (superficie de $1.000 \mathrm{~m}^{2}$ ). El agua recogida en ambos casos fue conducida a sus correspondientes registradores.

La variación temporal de la cantidad de agua edáfica fue medida en forma indirecta, empleando un dispositivo emisor de neutrones (modelo Troxler 3333). Para esta finalidad se insertaron verticalmente en el suelo tubos de acero de $45 \mathrm{~mm}$ de diámetro y $3 \mathrm{~m}$ de largo. La profundidad de $3 \mathrm{~m}$ estuvo determinada por la profundidad máxima que alcanzan las raíces según lo observado en las paredes de calicatas construidas para montar el experimento. Se distribuyeron 12 tubos al azar en el bosque y 3 en la pradera. La mayor cantidad de tubos en el primer caso se debe a una posible mayor variación en la distribución del agua edáfica producto de una cubierta vegetal más heterogénea. La sonda de neutrones fue calibrada según el método propuesto por Brechtel (1983). Debido a la difícil accesibilidad al área de estudio, las mediciones se realizaron una vez al mes durante un año.

Para conocer los niveles de saturación del perfil de suelo se determinó la capacidad de campo siguiendo el método propuesto por Van Der Merwe (1990). La capacidad de campo corresponde al agua retenida cuando las fuerzas opuestas del suelo se encuentran en equilibrio con el movimiento descendente de ella.

La escorrentía superficial (A) fue despreciada porque la pendiente del terreno es inferior a $2 \%$ y a la buena capacidad de infiltración del suelo (Schlatter et al. 2003). La percolación (PER) se consideró equivalente a la cantidad de agua que atraviesa un nivel de referencia prefijado a 3 $\mathrm{m}$ de profundidad. Esta agua fue recogida en 12 bandejas horizontales de 30 × $30 \mathrm{~cm}$. Para su instalación se hicieron previamente tres excavaciones profundas de $3 \times 3 \times 3,5 \mathrm{~m}$ que permitían introducir lateralmente las bandejas en cada una de las cuatro paredes del perfil. Cada bandeja estaba conectada mediante un tubo a un recipiente plástico con capacidad de 25 litros. A su vez, este recipiente estaba acoplado a un tubo de PVC de 2,54 cm de diámetro que alcanzaba a sobrepasar levemente la superficie del suelo. Las excavaciones se volvieron a cerrar para recuperar en la mejor forma posible las condiciones originales del suelo. Para cuantificar el agua acumulada en los recipientes se utilizó una bomba portátil, con la cual se extrajo el agua acumulada en ellos.

Para evaluar diferencias significativas en el consumo hídrico entre la pradera y el bosque de olivillo-roble se aplicó una prueba $t$ a los valores mensuales de evapotranspiración usando un nivel de confianza de 95\% (Dytham 2003).

\section{RESULTADOS}

La precipitación registrada en Valdivia durante el año de medición fue de $3.024 \mathrm{~mm}$, cantidad que superó en más de un $25 \%$ al promedio de la zona (cuadro 1 ). Se observó un fuerte contraste pluviométrico entre la estación de verano e invierno para el período de mediciones. Durante el verano, los meses de enero y febrero tuvieron un importante déficit pluviométrico y diciembre registró un exceso (cuadro 1). Los meses de marzo, abril y mayo fueron anormalmente lluviosos, mientras que agosto registró sólo la mitad del patrón promedio. Junio fue el mes más lluvioso del período de muestreo.

Cuadro 1. Precipitación mensual del período de medición y promedio de 25 años para Valdivia.

Monthly precipitation of the study period and average of 25 years in Valdivia city.

\begin{tabular}{lcc}
\hline Mes & $\begin{array}{c}\text { Año de estudio } \\
(\mathrm{mm})\end{array}$ & $\begin{array}{c}\text { Promedio } \\
(\mathrm{mm})\end{array}$ \\
\hline Septiembre 1992 & 218 & 199 \\
Octubre & 273 & 149 \\
Noviembre & 72 & 99 \\
Diciembre & 148 & 85 \\
Enero 1993 & 41 & 72 \\
Febrero & 12 & 64 \\
Marzo & 247 & 71 \\
Abril & 403 & 146 \\
Mayo & 464 & 379 \\
Junio & 502 & 369 \\
Julio & 489 & 408 \\
Agosto & 155 & 326 \\
\hline Total & 3.024 & 2.367 \\
\hline
\end{tabular}

Fuente: Instituto de Geociencias, Universidad Austral de Chile. 
Contenido de agua del suelo. En la pradera el contenido de agua promedio del perfil hasta los $3 \mathrm{~m}$ de profundidad fluctuó entre un $49 \%$ volumen para el mes de febrero a $58 \%$ para los meses de invierno (figura 2A). Durante el mes de febrero el porcentaje de humedad de la superficie $(0-50 \mathrm{~cm})$ fue de $31 \%$, mientras que en invierno este valor aumentó a un $52 \%$. La variación anual de la reserva de agua de todo el perfil fue equivalente a $268 \mathrm{~mm}$. En el bosque el contenido de agua promedio del perfil osciló entre $48 \%$ en febrero y un $60 \%$ en invierno (figura 2B). La mayor variación del contenido del agua edáfica también se registró en los primeros $50 \mathrm{~cm}$, con $33 \%$ volumen en febrero y $53 \%$ en junio. La mayor variación temporal del contenido de agua del perfil fue equivalente a $360 \mathrm{~mm}$.
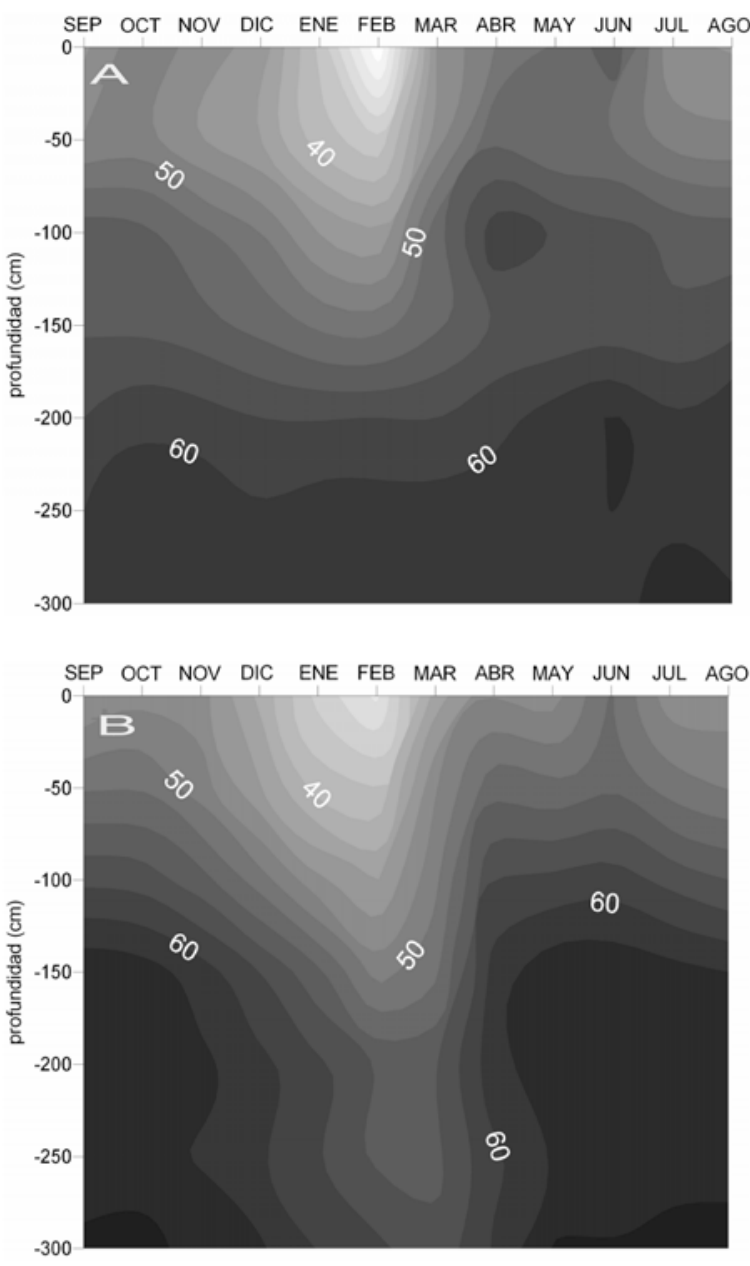

\section{戹 స్లు}

Figura 2. Variación espacial y temporal del contenido de humedad del suelo (\% volumen) de la pradera (A) y el bosque de roble-olivillo (B).

Spatial and temporal variation of soil water content $(\%$ volume) in the meadow (A) and the roble-olivillo forest (B).
Durante todo el año, la pradera mantuvo a profundidades mayores de $1 \mathrm{~m}$ una humedad cercana o superior a la capacidad de campo. A partir de octubre, en el primer metro de profundidad se registró un progresivo descenso de la cantidad del agua edáfica, la que se mantuvo hasta fines de febrero (figura 2A). Esta reducción se interrumpió abruptamente a principios de marzo, cuando las altas y anormales precipitaciones de este mes volvieron a recargar el suelo (figura $2 \mathrm{~A}$ ).

A diferencia de la pradera, el bosque experimentó variaciones de humedad en todo el perfil considerado (figura 2B). A principios de octubre, en el bosque se inició una reducción del contenido de agua, que duró hasta principios de marzo. Durante ese mes los dos primeros metros de suelo se comenzaron a saturar. La saturación de todo el perfil se logró durante el mes de abril (figura 2B).

Las mediciones obtenidas revelaron una baja variabilidad espacial en el agua edáfica en un determinado momento entre los puntos de muestreo en el bosque de roble-olivillo (cuadro 2). Es así como la desviación estándar varió desde 2,3 a $6,4 \%$ en el mes más seco (febrero) y de 0,8 a $4,9 \%$ en el mes más húmedo (julio). Esto refleja una mínima influencia de la heterogeneidad vegetacional del bosque de roble-olivillo en las variaciones del agua del suelo dentro de la parcela de estudio. En la pradera se observó una tendencia similar, pero con valores más bajos de desviación estándar (cuadro 3).

Los resultados además mostraron la ausencia de estratos impermeables que pudieran afectar el drenaje interno en los sitios estudiados. Esto se constata mediante un gradiente ascendente en los valores de capacidad de campo (CC) a medida que se avanza en profundidad. En particular, la CC promedio obtenida cada $50 \mathrm{~cm}$ en fue de 45,1; 48,3; 52,$2 ; 55,3 ; 57,1$ y $57,3 \%$ volumen.

Evapotranspiración. Los resultados revelaron diferencias significativas en los valores mensuales de evapotranspiración entre la pradera y el bosque de olivillo-roble $(\mathrm{t}=4,02$, $P<0,001)$. La evapotranspiración total de la pradera durante el período de estudio fue de $398 \mathrm{~mm}$ (cuadro 4), mientras que en el bosque de roble-olivillo este valor ascendió a $1.033 \mathrm{~mm}$, incluida la intercepción (cuadro 5). En la pradera sólo el 13\% de los aportes de agua fueron evapotranspirados. El bosque, por el contrario, consumió el 33\% de la precipitación total.

La tasa evapotranspirativa del bosque a través del año fluctuó entre un 1,2 $\mathrm{mm} /$ día durante los meses invernales y $3,8 \mathrm{~mm} /$ día en los estivales. Los meses de mayor demanda hídrica correspondieron a los de primavera y verano. Las mayores tasas evapotranspirativas ocurrieron en enero, con un valor promedio de $3,8 \mathrm{~mm} /$ día (cuadro 5). Durante el mes de febrero este valor descendió a 3,3 $\mathrm{mm} /$ día. Probablemente, la baja precipitación registrada en ese período redujo la disponibilidad de agua para la transpiración y las pérdidas por intercepción. Las pérdidas de agua por intercepción del bosque correspondieron a un $18 \%$ de la 


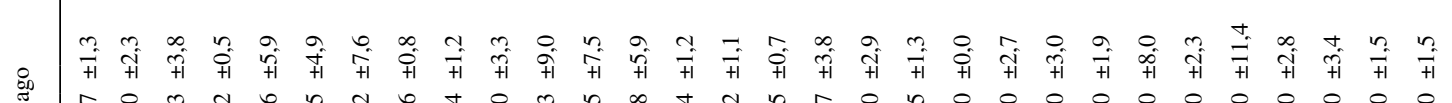

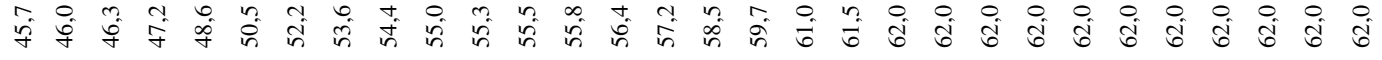

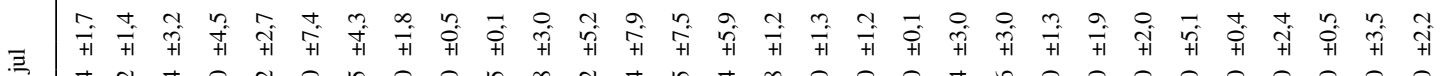

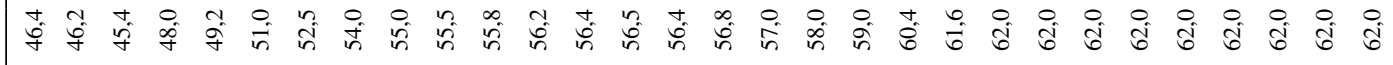

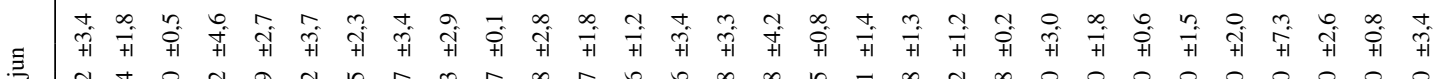

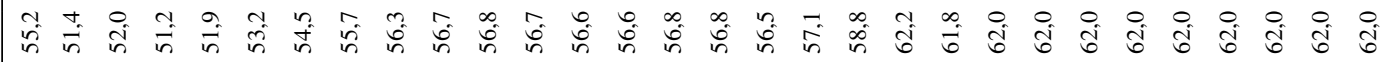

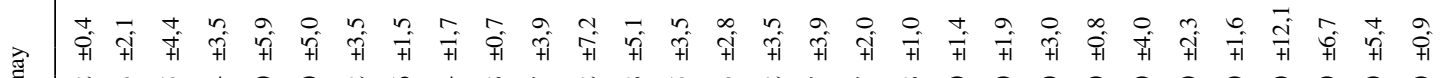

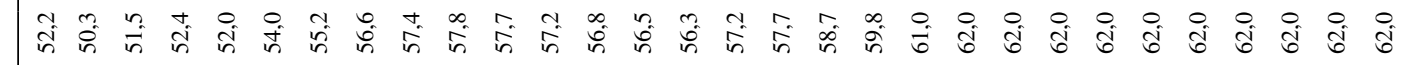

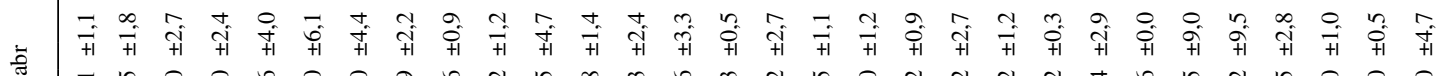

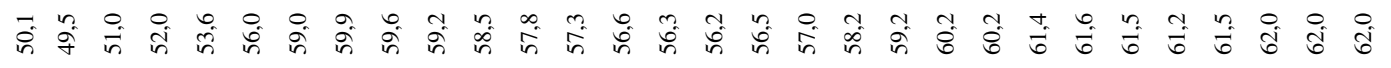

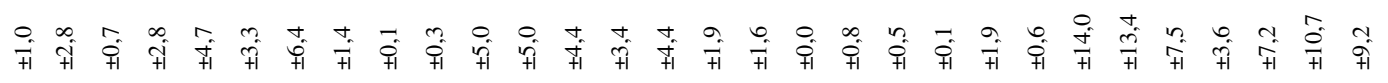

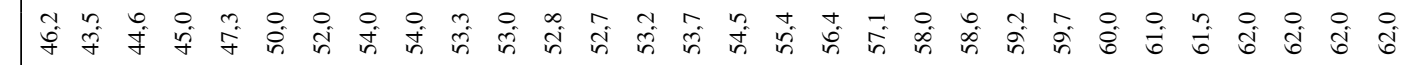

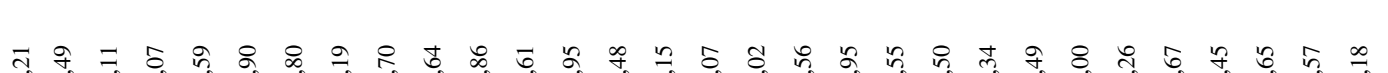

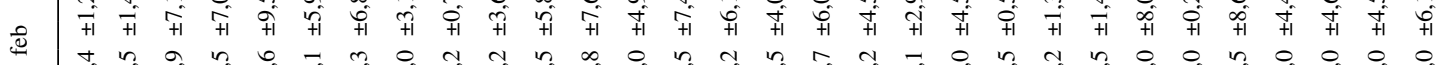

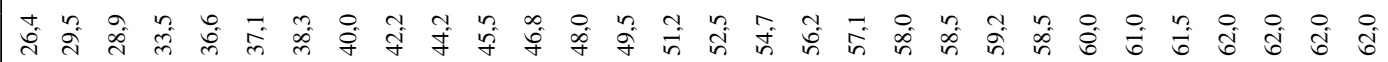

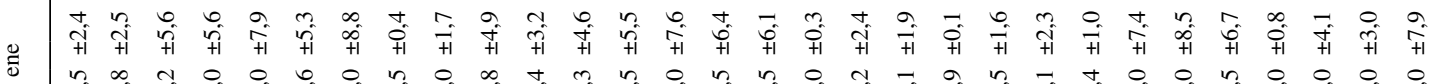

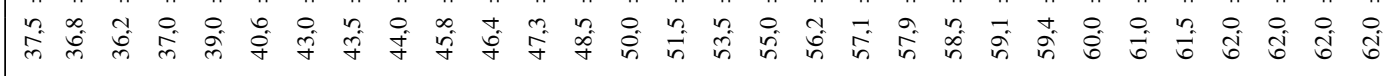

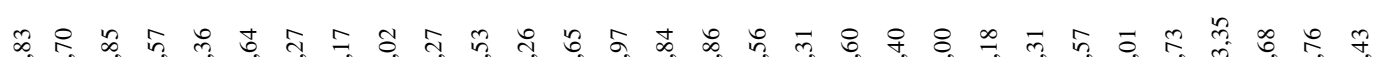

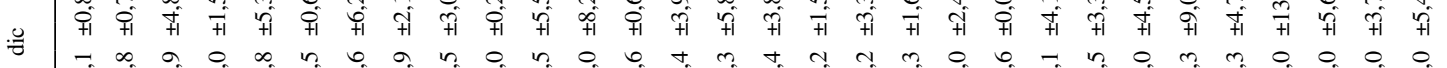

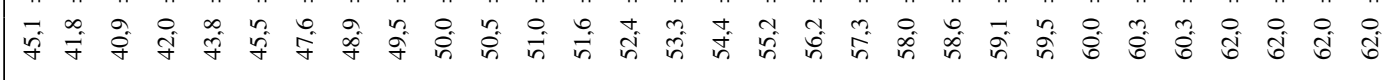

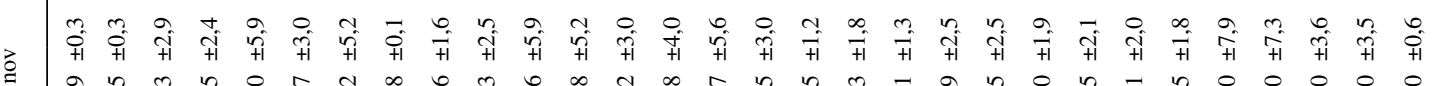

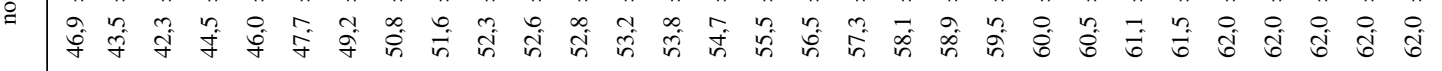

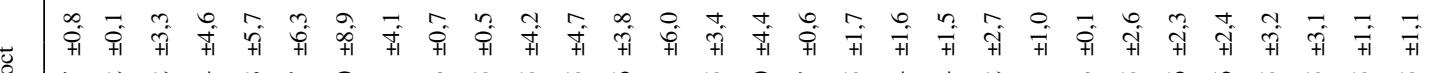

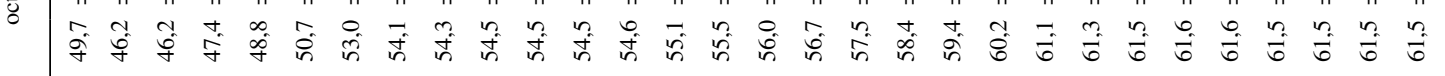

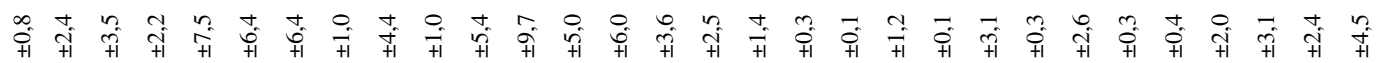

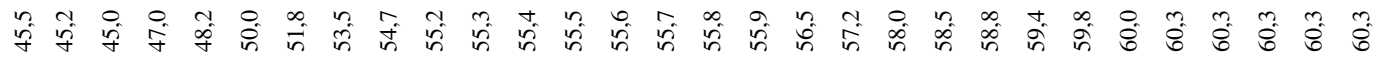


Cuadro 4. Variación temporal de la precipitación (PP), evapotranspiración (EVPT), evapotranspiración diaria (EVPT/día) y percolación (PER) de la pradera.

Temporal variation of precipitation (PP), evapotranspiration (EVPT), daily evapotranspiration (EVPT/día) and percolation (PER) in the meadow.

\begin{tabular}{lrccr}
\hline \multirow{2}{*}{ Mes } & \multicolumn{4}{c}{ Componente $(\mathrm{mm})$} \\
\cline { 2 - 5 } & PP & EVPT & EVPT/día & PER \\
\hline Sep & 148 & 21 & 0,7 & 126 \\
Oct & 355 & 28 & 0,7 & 302 \\
Nov & 175 & 27 & 1,0 & 188 \\
Dic & 107 & 45 & 1,3 & 108 \\
Ene & 76 & 97 & 2,8 & 110 \\
Feb & 33 & 78 & 2,8 & 0 \\
Mar & 243 & 60 & 1,5 & 14 \\
Abr & 300 & 20 & 0,8 & 185 \\
May & 430 & 1 & 0,0 & 426 \\
Jun & 527 & 2 & 0,1 & 527 \\
Jul & 502 & 2 & 0,1 & 538 \\
Ago & 204 & 17 & 0,5 & 179 \\
\hline Total & 3.100 & 398 & - & 2.703
\end{tabular}

Cuadro 5. Variación temporal de la precipitación (PP), intercepción (I), evapotranspiración (EVPT), evapotranspiración diaria (EVPT/día), evapotranspiración del suelo diaria (EVPTs/día) y percolación (PER) del bosque de roble-olivillo.

Temporal variation of precipitation (PP), interception (I), evapotranspiration (EVPT), daily evapotranspiration (EVPT/día), daily soil evapotranspiration (EVPTs/día) and percolation (PER) in the robleolivillo forest.

\begin{tabular}{lrrrrrr}
\hline & \multicolumn{5}{c}{ Componente (mm) } \\
\cline { 2 - 7 } & \multicolumn{1}{c}{ PP } & I & EVPT & EVPT/día & EVPTs/día & PER \\
\hline Sep & 148 & 37 & 50 & 1,6 & 0,4 & 98 \\
Oct & 355 & 89 & 117 & 3,1 & 0,7 & 235 \\
Nov & 175 & 38 & 65 & 2,5 & 1,0 & 163 \\
Dic & 107 & 35 & 121 & 3,4 & 2,4 & 125 \\
Ene & 76 & 25 & 130 & 3,8 & 3,1 & 0 \\
Feb & 33 & 19 & 94 & 3,3 & 2,7 & 0 \\
Mar & 243 & 33 & 140 & 3,6 & 2,8 & 0 \\
Abr & 300 & 60 & 80 & 3,3 & 0,8 & 24 \\
May & 430 & 70 & 71 & 2,2 & 0,0 & 320 \\
Jun & 527 & 51 & 53 & 1,8 & 0,1 & 453 \\
Jul & 502 & 43 & 44 & 1,2 & 0,1 & 488 \\
Ago & 204 & 51 & 68 & 2,0 & 0,5 & 155 \\
\hline Total & 3.100 & 551 & 1.033 & - & - & 2.061 \\
\hline
\end{tabular}

precipitación del período. Durante la estación invernal la evapotranspiración del bosque radicó principalmente en las pérdidas por intercepción. La transpiración y evaporación del suelo durante esta estación fueron mínimas.

La evapotranspiración del bosque y de la pradera volvió a ascender por la recarga de agua que tuvo el suelo debido a la altas precipitaciones $(243 \mathrm{~mm})$ registradas en marzo. Este ascenso en las precipitaciones también estuvo asociado a un aumento en las pérdidas de agua por intercepción. Estas precipitaciones permitieron saturar el perfil de la pradera y hasta los $2 \mathrm{~m}$ del bosque (figura 2).

Percolación. Los montos anuales de agua que percolaron por el nivel de referencia de $3 \mathrm{~m}$ de profundidad fueron de $2.703 \mathrm{~mm}$ en la pradera (cuadro 4) y de $2.061 \mathrm{~mm}$ en el bosque (cuadro 5). Estas cantidades correspondieron a un $87 \%$ y $66 \%$ de la precipitación total, respectivamente. Los mayores montos de percolación de ambos sitios se registraron durante los meses invernales, cuando todo el perfil edáfico estaba saturado. En la pradera la percolación se prolongó hasta los primeros días de enero. En el bosque, por el contrario, sólo hasta mediados de diciembre. Para la pradera, la percolación se reanudó rápidamente con la saturación de las primeras e intensas lluvias de marzo, mientras que esta situación en el bosque se alcanzó a mediados de abril.

\section{DISCUSIÓN}

A nivel superficial, la pradera presentó las mayores variaciones del contenido de humedad del suelo entre los meses de invierno y verano. Los resultados descritos concuerdan con experiencias realizadas en suelos profundos de California, donde cultivos herbáceos anuales manifestaron una tendencia similar en los primeros horizontes (Miller 1977). En el bosque estudiado también se observó la misma tendencia, pero estas variaciones se manifestaron a lo largo de todo el perfil de suelo examinado debido a la mayor extensión de las raíces de los árboles. Este mayor acceso al agua del suelo por la vegetación arbórea permite que los árboles estén expuestos a menos días con un suministro más limitado de humedad en comparación a una cubierta herbácea. Similares resultados fueron observados en un bosque de pinos y robles en España por López y Blanco (1978). En el sur de Chile, Huber y López (1993) también registraron un agotamiento del agua del suelo hasta los 280 $\mathrm{cm}$ de profundidad en una plantación de Pinus radiata D. Don, mientras que en una pradera y un área deforestada la disminución se observó sólo hasta los primeros $100 \mathrm{~cm}$. Grey (1989) y McCarthy et al. (1991) concluyeron que la profundidad de enraizamiento es el factor principal que determina la humedad disponible del perfil edáfico. Boden (1991) indica que la profundidad del sistema radical es un parámetro muy importante que se debe considerar al estudiar los regímenes hídricos del suelo. 
El monto de evapotranspiración referido a la pradera (398 mm) es inferior a lo determinado por Huber et al. (1985) en una cubierta vegetal similar ubicada en las cercanías con 501 mm/año. Huber y López (1993) estimaron un consumo aún mayor, con $625 \mathrm{~mm}$ y $509 \mathrm{~mm}$ durante dos años consecutivos para la misma pradera. Estas diferencias se deberían principalmente a las distintas pluviometrías registradas durante los correspondientes períodos de los estudios.

Los resultados también mostraron que el bosque de roble-olivillo consumió un $20 \%$ más de agua que la pradera, relación que se asemeja bastante con los resultados obtenidos por Johnson y Whitehead (1993) en el norte de Inglaterra. Estos autores confirman que una cuenca forestada con Fagus sylvatica L. tuvo un consumo hídrico que superó en un $21 \%$ a una cuenca con pastizales.

Tendencias similares en la tasa evapotranspirativa del bosque de roble-olivillo fueron obtenidas por McCarthy et al. (1991) al estudiar el balance hídrico en una cuenca forestada con Pinus taeda L. de 15 años de edad en Carolina del Norte. El estudio reveló tasas promedio que fluctuaron desde $1,4 \mathrm{~mm} /$ día para meses de invierno hasta $4,1 \mathrm{~mm} /$ día en verano.

Las pérdidas de agua por intercepción del bosque concuerdan con lo señalado por Pearce y Rowe (1979) y por Viville et al. (1993), que concluyen que el principal componente de la evapotranspiración es la evaporación del agua interceptada por la vegetación.

En el bosque de roble-olivillo la percolación de los primeros $3 \mathrm{~m}$ de profundidad correspondió a un $66 \%$ de la precipitación total. Un porcentaje inferior fue registrado en un bosque de alta montaña en Venezuela, en donde se determinó una percolación para el primer metro del suelo equivalente al $37 \%$ de la precipitación anual, pero para una zona que tiene una pluviometría anual de sólo 1.576 mm (Grimm y Fassbender 1981).

En la pradera estudiada, la percolación se prolongó hasta los primeros días de enero. En el bosque, por el contrario, sólo hasta mediados de diciembre. La menor percolación en el bosque durante el período primavera-verano se explica por una mayor profundidad de las raíces de los árboles, las que causan una disminución del contenido de humedad del perfil por más tiempo (Stogsdill et al. 1992).

Hibbert (1976) y Dons (1987) señalan que la producción hídrica puede ser aumentada al reducir la cubierta boscosa y arbustiva. Por lo tanto, la sustitución del bosque estudiado por una cubierta herbácea conduciría a un aumento de la cantidad de agua que percolaría por estos suelos. Dicho reemplazo derivaría de una reducción de las pérdidas por intercepción y por transpiración.

En el presente estudio, la pradera tuvo una mayor percolación durante todo el año. En el bosque, los montos de percolación se vieron disminuidos por la importante evapotranspiración y las pérdidas de agua por intercepción, que es la principal componente del consumo de agua de la cubierta boscosa. Las diferencias anteriormente men- cionadas concuerdan con lo señalado por Lee (1980), Papakyriakou y McCaughey (1991) y otros autores, al indicar que el mayor consumo hídrico y la menor cantidad de agua de percolación corresponde al bosque. Frente a esta situación, probablemente la tala rasa representa una buena estrategia para aumentar la producción hídrica (Troendle 1988). Esto se puede corroborar con los resultados de Huber y López (1993), en que después de la tala rasa de un bosque adulto de pino insigne, la evapotranspiración disminuyó y la percolación aumentó. Fernández et al. (2006) observaron que la tala rasa en una cuenca ubicada en el norte de España aumentó el caudal en un $73 \%$. A diferencia de la pradera o un terreno descubierto de vegetación, el bosque tiene un papel de regulador del agua: restituye menos agua pero de manera más regular. Esto hace que el bosque tenga una mayor importancia en regular los flujos en eventos de mayor cuantía (Detenbeck et al. 2005, Giertz et al. 2005). Al contrario, una pradera ubicada en zonas con pendiente puede producir más agua y con una respuesta más rápida e intensa a variaciones en precipitaciones, lo cual está asociado a un mayor arrastre de sedimentos y, por ende, una menor calidad del agua (Ward y Trimble 2004).

Los valores de los componentes del balance hídrico del presente estudio fueron registrados durante un período en el cual la precipitación fue más de un $25 \%$ superior al promedio de los últimos 25 años. Este superávit de precipitación estaría garantizando un consumo máximo de agua por evapotranspiración del bosque de roble-olivillo y la pradera en el período de medición. Es probable que los altos aportes de precipitación hayan permitido disponer de toda el agua necesaria para los requerimientos hídricos del bosque, necesarios para su desarrollo. En caso de años secos o con una precipitación menor a la de un año promedio, es probable que los componentes del balance hídrico pudieran presentar valores inferiores tanto en la pradera como en el bosque.

$\mathrm{Si}$ bien el estudio puede presentar limitaciones en capturar la variabilidad de la humedad del suelo a través de mediciones realizadas una vez al mes, los resultados demuestran que dicha frecuencia da una aproximación válida de la variación temporal del agua edáfica en un bosque de roble-olivillo y pradera a lo largo de un período de un año.

\section{CONCLUSIONES}

La tendencia temporal del contenido de humedad del suelo tuvo una marcada fluctuación estacional, tanto en el bosque como en la pradera. Si bien en el bosque las variaciones espaciales del agua edáfica abarcaron la totalidad del perfil analizado $(3 \mathrm{~m})$, en la pradera los primeros 100 $\mathrm{cm}$ de suelo resultaron más fuertemente desecados.

La evapotranspiración total anual del bosque duplicó a la registrada en la pradera. Los aportes de agua por perco- 
lación fueron $20 \%$ mayores en la pradera que en el bosque. Dicha diferencia se debe principalmente a la intercepción de las precipitaciones ejercida por las copas de los árboles. En ambos sitios se registra un aumento substancial en la percolación durante el otoño y el invierno.

La variación temporal de los componentes del balance hídrico del bosque y la pradera se debió fundamentalmente a la distribución de las precipitaciones, a las condiciones climáticas, a las características aéreas de la vegetación y a la morfología y profundidad del sistema radicular. Se puede concluir que el reemplazo de un bosque por una cubierta herbácea inducirá a cambios importantes en los aportes de agua que llegarán al suelo mineral, en la distribución espacial y temporal del contenido de agua del suelo y en la profundidad y en la cantidad de agua involucrada en la evapotranspiración y percolación.

Los resultados presentados en este estudio deben ser asociados a las condiciones de precipitación registradas durante el año de medición, las cuales fueron superiores a un año promedio. Comparaciones con otros estudios deberían ser hechas con cautela, considerando que los valores aquí presentados podrían equivaler a valores máximos de consumo hídrico que este tipo de bosque podría requerir.

\section{REFERENCIAS}

Aussenac G, C Boulangeat. 1980. Interception des précipitations et évapotranspiration réelle dans des peoplements de feuillu (Fagus sylvatica L.) et de résineux (Pseudotsuga menziesii (Mirb) Franco). Annales des Sciences Forestières 37(2): 91-107.

Boden D. 1991. The relationship between soil water status, rainfall and the growth of Eucalyptus grandis. South African Forestry Journal 156: 49-55.

Brechtel HM. 1983. Probleme bein Einsatz von Neutronensonde im Rahmen Hydrologischer Messprogramme. Deutscher Verband für Wasserwirtschaft und Kulturbau. Alemania, Verlag Paul Parey. 301 p.

Crockford RH, DP Richardson. 1990. Partitioning of rainfall in a eucalypt forest and pine plantation in southeastern Australia: IV. The relationship of interception and canopy storage capacity, the interception of these forests, and the effect on interception of thinning the pine plantation. Hydrological Processes 4(2): 169-198.

Daniel TW, JA Helms, FS Baker. 1982. Principios de Silvicultura. México. Primera Edición. Mc Graw-Hill. 492 p.

Detenbeck N, V Brady, D Taylor, V Snarski, S Batterman. 2005. Relationship of stream flow regime in the western Lake Superior basin to watershed type characteristics. Journal of Hydrology 309: 258-276.

Dons A. 1987. Hydrology and sediment regimen of a pasture, native forest and pine forest catchments in the Central North Island, New Zealand. New Zealand Journal of Forestry Science 17 (2/3): 161-178.

Echeverría C, D Coomes, J Salas, JM Rey-Benayas, A Lara, A Newton. 2006. Rapid deforestation and fragmentation of Chilean Temperate Forests. Biological Conservation 130: 481-494.

Fernández C, J Vega, J Gras, T Fonturbel. 2006. Changes in water yield after a sequence of perturbations and forest management practices in a Eucalyptus globulus Labill. Watershed in Northern Spain. Forest Ecology and Management 234: 275-281.

Feller MC. 1981. Water balance in Eucalyptus regnans, E. obliqua, and Pinus radiata forests in Victoria. Australian Forestry 44(3): 153-161.

Giertz S, B Jungue, B Diekkrüguer. 2005. Assessing the effects of land use change on soil physical properties and hydrological processes in the sub-humid tropical environment of West Africa. Physics and Chemistry of the Earth 30: 485-496.

Grey DC. 1989. Site requirements of Pinus radiata: A review. South African Forestry Journal 148: 23-26.

Grimm U, H Fassbender. 1981. Ciclos biogeoquímicos en un ecosistema forestal de los Andes Occidentales de Venezuela III: ciclo hidrológico y translocación de elementos químicos con el agua. Turrialba 31(2): 89-99.

Hewlett J, W Nutter. 1969. An outline of forest hydrology. Georgia. University of Georgia Press. 137 p.

Hibbert A. 1976. Percolation and stream flow in range and forest lands. Watershed management on range and forest lands. In Proceedings of the Fifth Workshop of the United States/Australia Rangelands Panel Boise, Idaho, June 15-22, 1975. Utah, United States of America, Utah Water Research Laboratory, Utah State University. p. 61-72.

Huber A. 1970. Diez años de observaciones climáticas en la Estación Teja-Valdivia, Chile. 1960-1969. Valdivia, Chile, Facultad de Ciencias Naturales y Matemáticas. Universidad Austral de Chile. 60 p.

Huber A, C Oyarzún, A Ellies. 1985. Balance hídrico en tres plantaciones de Pinus radiata. II: Humedad del suelo y evapotranspiración. Bosque 6(2): 74-82.

Huber A, C Oyarzún, 1990. Variaciones anuales en la precipitación, escurrimiento e intercepción en un bosque de Pinus radiata. Turrialba 40(4): 503-508.

Huber A, D López. 1993. Cambios en el balance hídrico provocados por la tala rasa de un rodal adulto de Pinus radiata (D. Don). Bosque 14(2): 11-18.

IREN (Instituto Nacional de Investigación de Recursos Naturales, CL), CORFO (Corporación de Fomento de la Producción, CL), UACh (Universidad Austral de Chile, CL). 1978. Estudio de suelos de la Provincia de Valdivia. Instituto Nacional de Investigación de Recursos Naturales. Corporación de Fomento de la Producción. Universidad Austral de Chile. Santiago, Chile. 178 p.

Johnson R, P Whitehead. 1993. An introduction to the research in the Balquhidder experimental catchments. Journal of Hydrology 145: 231-238.

Knoche D. 2005. Effects of stand conversion by thinning and underplanting on water and element fluxes of a pine ecosystem (P. sylvestris L.) on lignite mine spoil. Forest Ecology and Management 212: 214-220.

Lee R. 1980. Forest Hydrology. New York, United States of America. Columbia University Press. 349 p.

López C, C Blanco. 1976. Hidrología Forestal. Primera Parte. Madrid, España. Escuela Técnica Superior de Ingenieros de Montes. 387 p. 
López C, C Blanco. 1978. Hidrología Forestal. Segunda Parte. Madrid, España. Escuela Técnica Superior de Ingenieros de Montes. 134 p.

McCarthy EJ, RW Skaggs, P Farnum. 1991. Experimental determination of the hydrologic components of a drained forest watershed. Journal Series of Department of Biological and Agricultural Engineering 118(2): 242-255.

Miller D. 1977. Water at the Surface of the Earth. An Introduction to Ecosystem Hydrodynamics. International Geophysics series. Nueva York, Estados Unidos de América. Academic Press. 557 p.

Neira E. 2005. Producción de agua en dos microcuencas de la Cordillera de Los Andes (San Pablo de Tregua) con diferente cobertura de renovales de Nothofagus. Tesis Magíster en Ciencias, Mención Recursos Forestales. Valdivia, Chile. Facultad de Ciencias Forestales, Universidad Austral de Chile. 93 p.

Papakyriakou TN, JH McCaughey. 1991. An evaluation of the water balance technique for the estimation of evapotranspiration for a mixed forest. Canadian Journal of Forest Research 21: 1622-1631.

Pearce AJ, LK Rowe. 1979. Forest management effects on interception, evaporation, and water yield. Journal of Hydrology 18(2): 73-87.

Schlatter JE, R Grez, V Gerding. 2003. Manual para el reconocimiento de suelos. Valdivia, Chile. Universidad Austral de Chile. 114 p.

Spurr S, B Barnes. 1982. Ecología Forestal. México. AGT Editor. 690 p.

Steubing L, C Ramírez, M Alberdi. 1979. Artenzusammensetzung, Lichtgenuss und Energiegehalt der Krautschicht des val- divianischen Regenwaldes bei St Martin. Vegetatio 39(1): 25-33.

Stogsdill WR, RF Wittwer, TC Hennessey, PM Dougherty. 1992. Water use in thinned loblolly pine plantations. Forest Ecology and Management 50: 233- 245.

Thanapakpawin P, J Richey, D Thomas, S Rodda, B Campbell, M Logsdon. 2006. Effects of land use change on the hydrologic regime of the Mae Chaem river basin, NW Thailand. Journal of Hydrology 334: 215-230.

Troendle CA. 1988. Effect of partial cutting and thinning on the water balance of the subalpine forest. In Proceedings future forests of the mountain best: Stand culture symposium 1986 September 29 -October 3. Ogden, Utah, Estados Unidos de América. US Department of Agriculture, Forest Service, Intermountain Research Station. p. 108-116.

Van Der Merwe L. 1990. A technique for producing soil water retention curves. South African Forestry Journal 155: 27-29.

Viville D, P Biron, A Granier, E Dambrine, A Probst. 1993. Interception in a mountainous declining spruce stand in the Strengbach catchment (Vosges, France). Journal of Hydrology 144: 273-282.

Ward A, S Trimble. 2004. Environmental hydrology. United States of America. Lewis Publishers. 475 p.

Weinberger P, R Binsack. 1970. Zur Entstehung und Verbreitung der Aschenböden in Südchile. Der Tropenlandwirt 72: 51-71.

Zimmermann B, H Elsenbeer, J De Moraes. 2006. The influence of land-use changes on soil hydraulic properties: Implications for runoff generation. Forest Ecology and Management 222: 29-38. 